\title{
The Influence of Static Magnetic Field on Silica Gel Free Interphase Energy
}

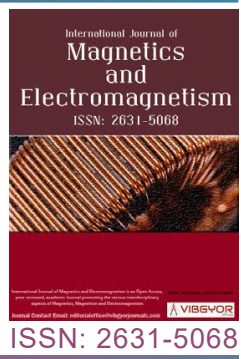

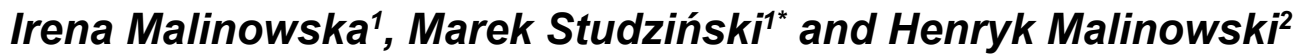

${ }^{1}$ Faculty of Chemistry, Institute of Chemistry, Chair of Physical Chemistry, Maria Curie-Skłodowska University, Poland

${ }^{2}$ Joint Institute of Nuclear Research, Vexler and Baldin Laboratory of High Energy Physics, Russia

\begin{abstract}
In presented paper influence of external, homogeneous magnetic field (one of the most common fields creating the universe) on interfacial Gibbs energy and its component were investigated. Basing on van Oss, et al. approach, components of Gibbs' interfacial energy of porous silica gel, using thin layer wicking method, were determined without and in homogenous magnetic field with induction $0,44 \mathrm{~T}$. Compared results obtained inside and outside of strong magnetic field shows significant differences between experimental results obtained in 0,4T uniform static magnetic field and outside it. It proves that the field influences on free interphase energy value of porous silica gel.
\end{abstract}

\section{Keywords}

Static magnetic field, Free interphase energy, Thin layer wicking

\begin{abstract}
Abbreviations
$\gamma$ : Free interfacial energy; $\gamma^{A B}$ : Polar component of free interfacial energy (acid-base component); $\nu^{\lfloor W}$ : Apolar component of free interfacial energy (Lifshitz-van der Waals component); $v^{-}$: Electron donor parameter of polar component of free interfacial energy; $v^{+}$: Electron acceptor parameter of polar component of free interfacial energy; $\gamma^{d}$ : London parameter of apolar component of free interfacial energy; $\gamma^{p}$ : Debye parameter of apolar component of free interfacial energy; $\gamma^{i}$ : Keesom parameter of apolar component of free interfacial energy; $\gamma_{L}$ : Free interfacial energy of liquid; $\gamma_{s}$ : Free interfacial energy of solid; $\gamma_{S L}$ : Solid-liquid free interfacial energy; $\Delta G_{12}^{a}(A B)$ : Acid-base (polar) component of Gibbs interfacial energy between two phases; $\Delta G_{12}^{a}(L W)$ : Lifshitz van der Waals (apolar) component of Gibbs interfacial energy between two phases; $\Delta G$ : Free interfacial energy during porous layer penetration by liquid; $\Delta G_{b}$ : Free interfacial energy calculated from Washburn equation for dry layers; $\Delta G_{p}$ : Free interfacial energy calculated from Washburn equations for conditioned layers; $R$ : Effective radius of porous bed (effective radius of interparticle capillaries); $\eta$ : Liquid viscosity; $t$ : Time of penetration porous solid layer by liquid; $x$ : Distance of penetration porous solid layer in time $t$.
\end{abstract}

\footnotetext{
*Corresponding author: Marek Studziński, Faculty of Chemistry, Institute of Chemistry, Chair of Physical Chemistry, Plac Marii Curie-Skłodwskiej 3 pok. 223, 20-031 Lublin, Poland, Tel: +48815377748

Accepted: December 08, 2020; Published: December 10, 2020

Copyright: (C) 2020 Malinowska I, et al. This is an open-access article distributed under the terms of the Creative Commons Attribution License, which permits unrestricted use, distribution, and reproduction in any medium, provided the original author and source are credited.
}

Malinowska I, et al. Int J Magnetics Electromagnetism 2020, 6:032






\section{Introduction}

Magnetic field is one of the most common fields creating the universe. As it was proven before [1-3] its presence influences on some processes taking place in the interfacial area, but investigations consisting influence of static magnetic field on change of values of components of free surface energy, according to our knowledge, have never been performed.

The reason of existing all interface phenomena are unbalanced intermolecular forces appearing on the border of two phases and in the layers contacting them, which is named the interfacial tension, or in case where one of the phases is gas the surface tension. Free surface energy or more precisely Gibbs interfacial energy, is the function which quantitatively describes the interfacial tension phenomena. It can be defined as work needed to create new interface of given area between two phases. Thus, the ability to manipulate its value by applying external physical field can revolutionize many areas of human activities such as application or remotely controlled drug and gene delivery systems, chromatographic separation of inseparable (until now) compounds of plant extracts or even invention? of molecule size transistors, creating molecular thickness coatings and many others.

The aim of this work is to determinate of free surface Gibbs' energy components in different interfacial systems of silica gel by presence of external uniform static magnetic field.

\section{Calculating free interfacial energy}

At present, there are two approaches to determining the interfacial Gibbs energy. One of them claims that it is a sum of intermolecular interactions of various types and those components has crucial influence on determining it. The second, implies that it is sum of free interfacial energies of contacting phases. Representation of first type approach is the way of determination of free surface energy proposed in 1962 by Fowkes [4] who claimed that the total surface energy can be calculated by summing of geometrical means of every element contacting on the border of two phases.

The other approach is represented by van Oss, et al. [5-7], who assumed that free interfacial energy can be presented by the sum of polar and apolar interactions:

$$
\gamma=\gamma^{A B}+\gamma^{L W}
$$

Polar interactions, in most cases, are the result of creation hydrogen bonds among neighbouring molecules, and can be described as:

$$
\gamma^{A B}=2\left(\gamma^{+} \gamma^{-}\right)^{\frac{1}{2}}
$$

Taking into consideration models of apolar interactions proposed by Girifalco and Good [8] and Lifshitz theory [9], one can assume that apolar component of free interfacial energy is equal to:

$$
\gamma^{d}+\gamma^{p}+\gamma^{i}=\gamma^{L W}
$$

Because of the fact that dipole - dipole and dipole - induced dipole interactions participation in total free interfacial energy is smaller than $2 \%$ (as the result of mutual saturation of those) and can be omitted. Thus, main part of apolar interactions comes from London's dispersion interactions [7].

Maintaining under consideration rules of Small $[10]$ and Kolmann $[11,12]$ concerning short range interfacial forces, describing acid-base component of free adhesion between phases 1 and 2 as:

$$
\Delta G_{12}^{a}(A B)=-2\left(\gamma_{1}^{+} \gamma_{2}^{-}\right)^{1 / 2}-2\left(\gamma_{1}^{-} \gamma_{2}^{+}\right)^{1 / 2}
$$

also, if one know that:

$$
\Delta G_{12}^{a}=\Delta G_{12}^{a}(L W)+\Delta G_{12}^{a}(A B)
$$

The final expression describing interfacial Gibbs energy can be written as:

$$
\Delta G_{12}^{a}(A B)=-2\left(\gamma_{1}^{L W} \gamma_{2}^{L W}\right)^{1 / 2}-2\left(\gamma_{1}^{+} \gamma_{2}^{-}\right)^{1 / 2}-2\left(\gamma_{1}^{-} \gamma_{2}^{+}\right)^{1 / 2}
$$

On the basis of presented considerations, van Oss, et al. proposed equation describing interfacial interactions between two liquid phases and in solid - liquid system which was written as follows:

$\gamma_{S L}=\left(\sqrt{\gamma_{S}^{L W}}+\sqrt{\gamma_{L}^{L W}}\right)^{2}+2\left(\sqrt{\gamma_{S}^{+} \gamma_{S}^{-}}+\sqrt{\gamma_{L}^{+} \gamma_{L}^{-}}-\sqrt{\gamma_{S}^{+} \gamma_{L}^{-}}-\sqrt{\gamma_{S}^{-} \gamma_{L}^{+}}\right)$

Equation 7 have been used many times for calculating free interfacial energy by van Oss and his co-workers, and also for experimental determination of free surface energy for experimental determination of free surface energy giving satisfactory results.

\section{"Thin Layer Wicking" method of free interfa- cial energy determination}

In order to better understand phenomena taking place in the interfacial area between liquid and 


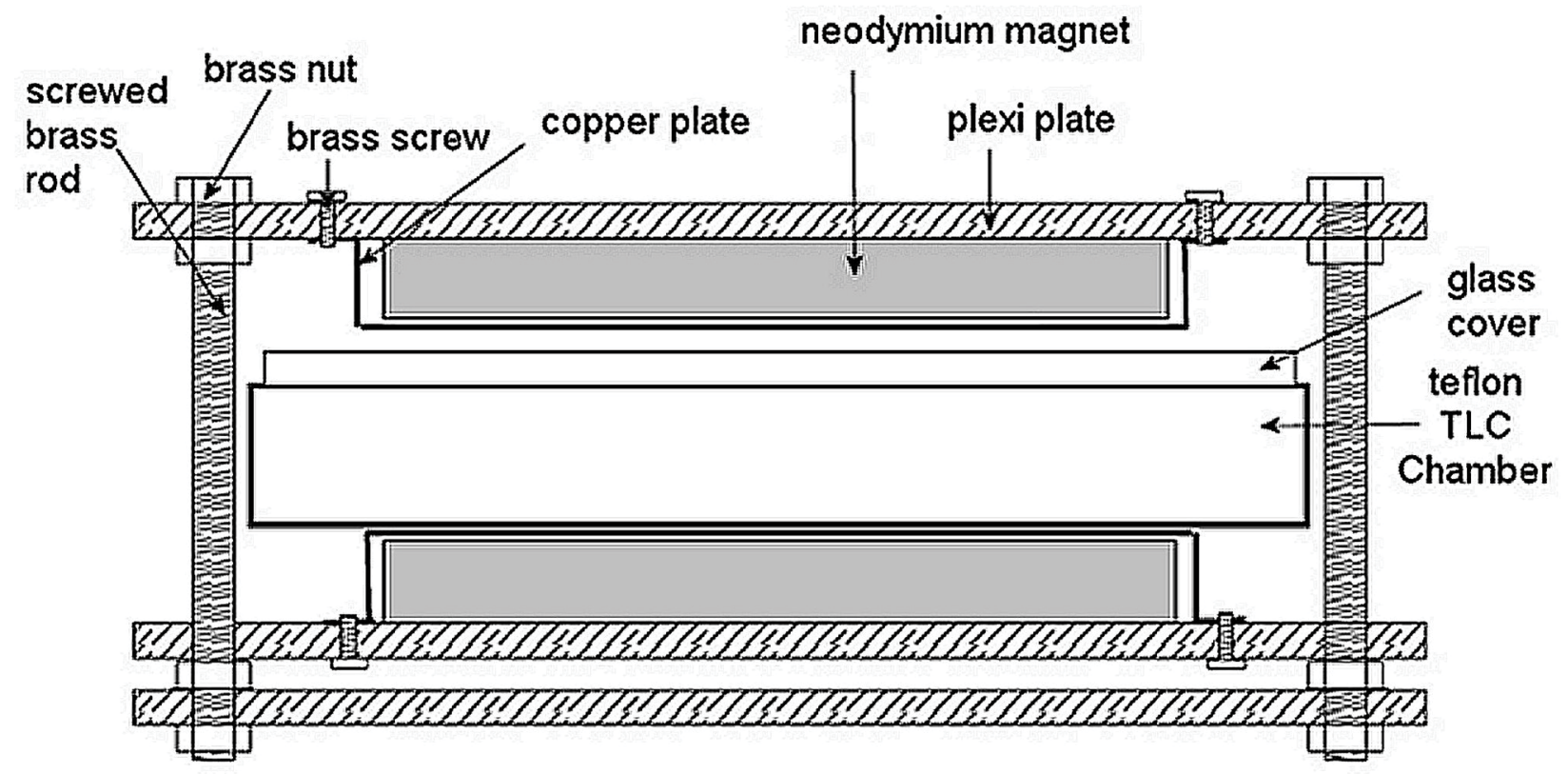

Figure 1: Scheme of device for liquid velocity on porous layer determination in magnetic field.

solid phase, components of free surface energy were determined according to Van Oss, et al. approach using "thin layer wicking" method. As it was presented before [13-17] modified Washburn equation [18-20]:

$$
x^{2}=\frac{R t}{2 \eta} \Delta G
$$

can be applied for free surface energy determination.

$x$ and $t$ values are obtained by measurement of time of penetration of given solvent of given distance $(\mathrm{x})$ of porous bed.

\section{Experimental}

The basis for description of static magnetic field influence on different interfacial phenomena eg. mixture separation on porous layer is to determine and describe way the field changes interactions and properties of surface.

Measuring the penetration velocity of given liquids on porous layer of solid is one of the most important experiments leading to determination of free surface energy of those solids. Similarity of that experiment to chromatogram development in thin layer chromatography, induces the fact that method introduced by Van Oss, et al. [5-7] is the best solution for free surface energy of sorbents. The use of commercial TLC plates covered silica gel improves the reliability and repeatability of experiments presented in this work.

\section{Materials and methods}

All solvents (gradient grade) and TLC plates (TLC $\mathrm{SiO}_{2}$ without fluorescent indicator) used in experiment were delivered by Merck (Darmstadt, Germany).

Experiments in static magnetic field were performed using a pair of permanent neodymium magnets (delivered by ENES, Warsaw) mounted in specially constructed device which scheme is presented on Figure 1.

Inductivity of uniform static magnetic field induced in the device was about 0.44 Tesla. For further technical description of the equipment please refer to our earlier works (e.g. [1-3]).

Penetration time of chosen solvent was measured on the distance $10 \mathrm{~cm}$ with step of $1 \mathrm{~cm}$ (with exception water). In case experiment carried out on conditioned by given solvent sorbent beds, silica layers were preconditioned, right before penetration velocity measurements, for 30 minutes in TLC chamber which interior was saturated the vapours of solvent. Such time of plate preconditioning gives good base to assume that the surface of silica gel was uniformly covered by the film of given solvent regarding its volatility is one of the most common fields creating the universe is one of the most common fields creating the universe. All experiments were performed at room temperature $\left(20 \pm 1^{\circ} \mathrm{C}\right)$. All measurements were repeated at least 3 times. 
All blunders were rejected. All presented calculations were carried out on the basis of mean values calculated from 3 consecutive measurements.

\section{Effective radius of interparticle capillaries (R) determination}

The value of effective radius of interparticle capillaries informs about the penetration ability of sorbent bed by solvent used for determination of that parameter. $R$ is determined on the basis of velocity of wetting fronts of two or three different solvents used in experiment. Effective radius depends from type of liquid used in experiment [21], thus comparing of $R$ values obtained in uniform, parallel to plate plane magnetic field and outside it allows for gathering data about the field influence on given solvents. Effective radius of interparticle capillaries can be determined measuring of velocity of penetration of liquid in porous layer, preserving following conditions:

Liquid should totally wet the surface of solid (in this case $\Delta G=\gamma_{L}$ ),

The surface of solid should be covered by duplex film of penetrating liquid (or the film should form in front of penetrating liquid).
Given conditions are fulfilled for liquid aliphatic hydrocarbons, and in order to create duplex film sorbent layer is conditioned in corresponding vapours before beginning of time of penetration measurement.

In considered system, equation 8 transforms in eq. 9

$$
R=\frac{2 \eta x^{2}}{t \gamma_{L}}
$$

Which is useful for, $R$ value calculation. Theoretically, in this particular case velocity of liquid penetration does not depend from properties of solid surface, but from properties of liquid used in experiment.

In order to determine $R$ value time of migration of three $n$-alkanes on silica gel layers have been measured, in magnetic field and outside one simultaneously. Obtained results are presented on Figure 2.

According eq. 8 and on the basis of experimental results linear correlation between time of migration and square of migration distance can be confirmed. Moreover, differences between migration velocity of corresponding $n$-alkanes in the magnetic field and outside it can be observed.

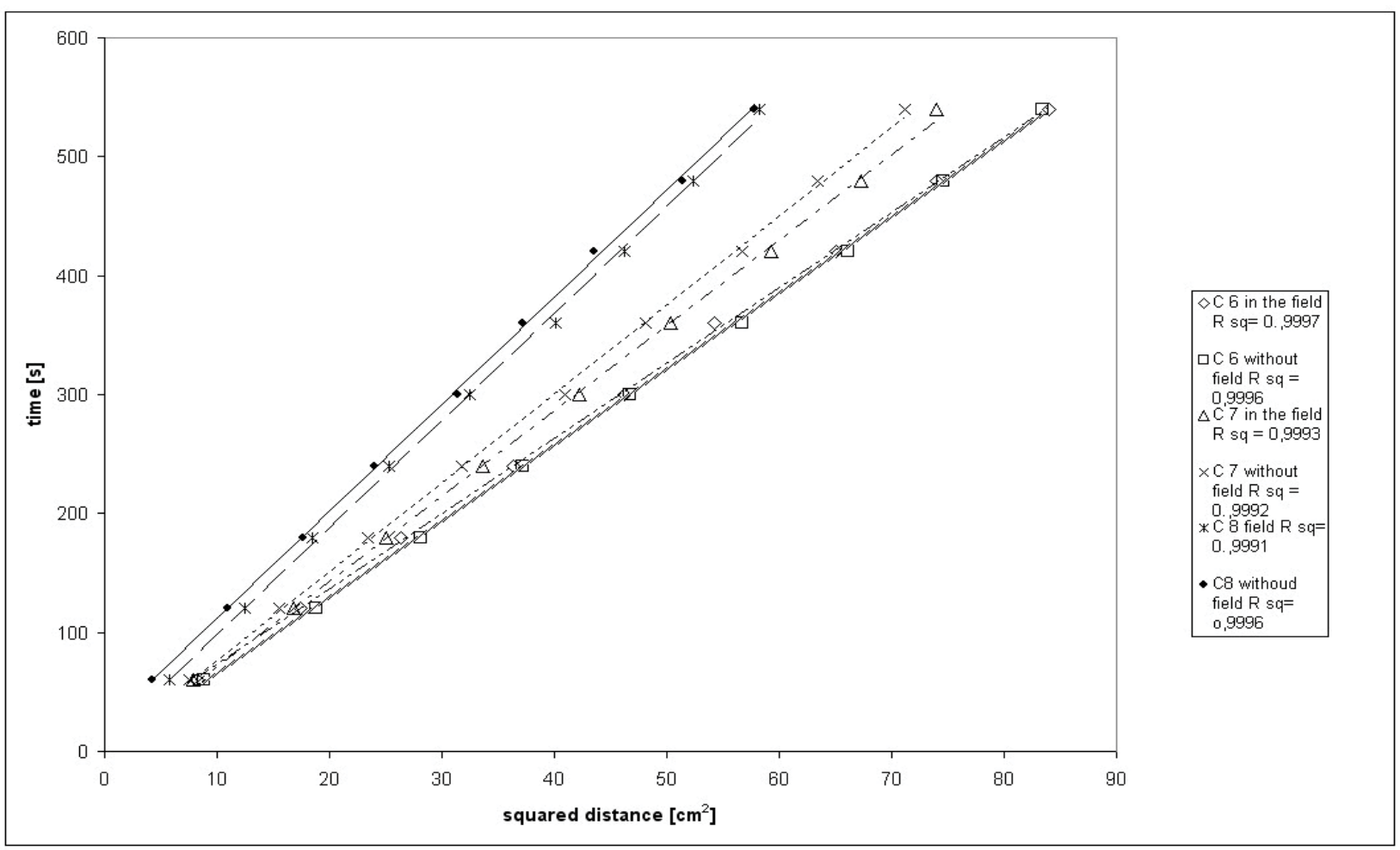

Figure 2: Dependence of migration time of $n$-alkanes vs. square of migration distance on conditioned layers of silica gel in magnetic field and outside it (n-hexane (C-6), n-heptane (C-7) and n-octane (C-8). 




Figure 3: $R$ values obtained for $n$-hexane (C6), $n$-heptane (C7) and n-octane (C8) in magnetic field and outside it.

Obtained values of effective radii of interparticle capillaries in the magnetic field and outside it for thee aliphatic hydrocarbons are presented on Figure 3.

As it can be seen on Figure 3, in uniform perpendicular to direction of liquid migration magnetic field $R$ values are different from those obtained in experiment outside the field. It is smaller in case of $\mathrm{n}$-hexane but bigger in case of two other $\mathrm{n}$-alkanes used in this study. That change provides the change of penetration ability of particular liquid. Decrease of effective radius of interparticle capillaries is probably caused by configuration change of $n$-hexane molecule what allows it to penetrate smaller pores of stationary phase ( $n$-hexane chain may be straightened in magnetic field $[22,23])$. Increase of $R$ in case of $n$-heptane and $n$-octane may be also result of molecule configuration change, but the modification is different than in a case of $n$-hexane what makes it difficult to infiltrate smaller pores of stationary phase.

\section{Influence of magnetic field on apolar compo- nent of free interfacial energy of silica gel}

If the liquid fully wets porous surface of solid, however surface of solid in front of solvent front is dry (there is no duplex film on it) it is known from earlier investigations that spread work (difference between adhesion and cohesion work) is equal to free enthalpy change. When the effective radius interparticle capillaries are known, measurement of mobile phase front velocity allows to determine of $\Delta G_{b}$ values.

Calculated $\Delta G_{b}$ values according to eq 8 of silica for investigated hydrocarbons are depicted on Figure 4.

The biggest differences of spread work between experiments carried out in magnetic field and outside it in case of $n$-hexane were observed. There are bibliographic sources describing influence of magnetic field on physical properties of various bodies can change some physicochemical properties [2426]. It was observed, that the changes does not depend linearly from induction of the field. They may reach maximum for given inductivity, and further increase of field inductivity causes decease of those quantities. Thus, the most probable explanation of obtained result is hypothesis that used in experiment magnetic field was suitable for produce observed effect in case of n-hexane, contrary to two other researched aliphatic hydrocarbons.

Because mentioned liquids interact with the surface of solid phase only by Lifshic-Van der Waals forces, so the whole free interfacial energy of liquid can be considered as apolar component of free surface energy. Solving the equation (9) with experimental data allows to calculate apolar component for free surface energy of solid (Figure 5). 


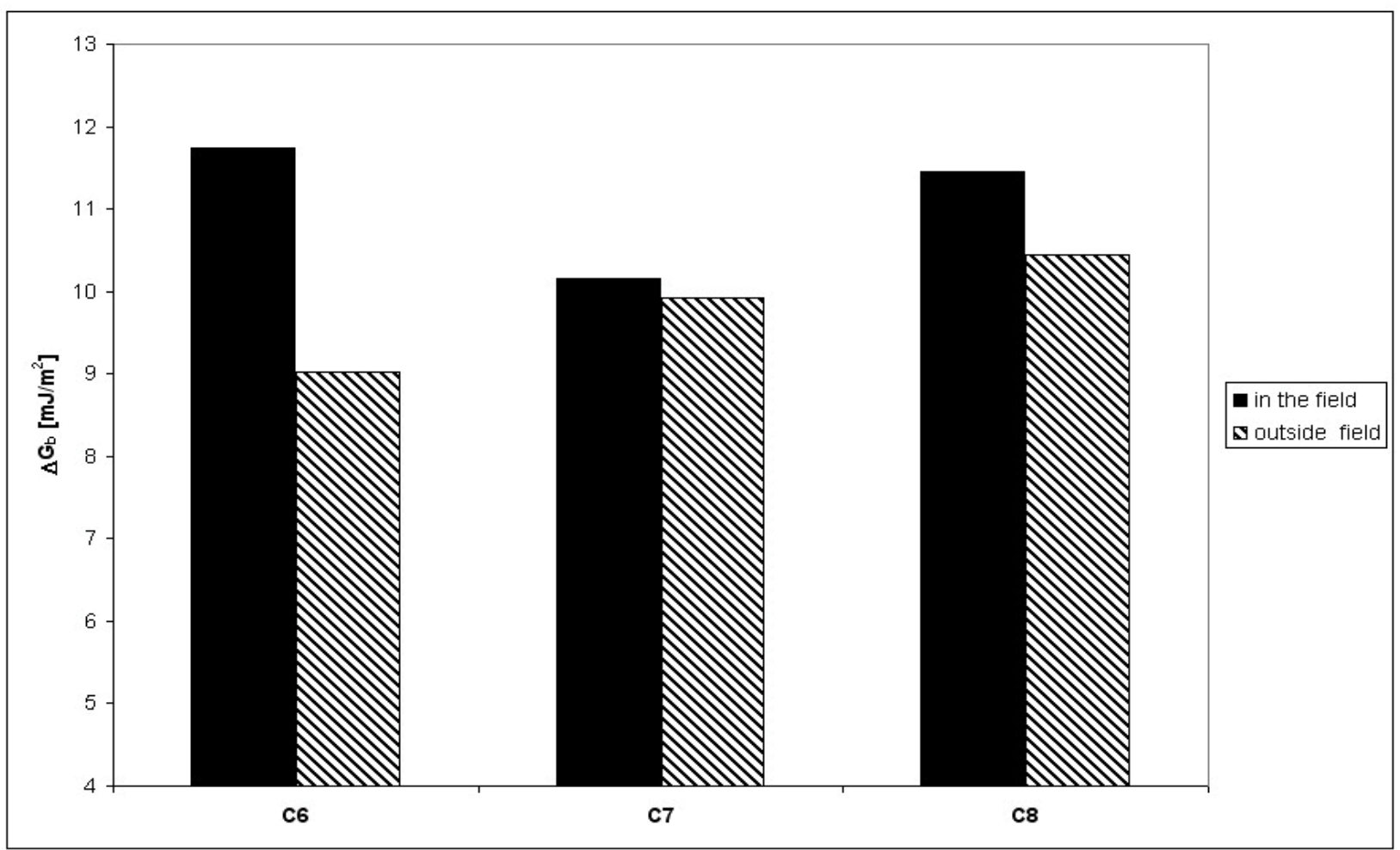

Figure 4: Comparison of $\Delta G_{b}$ values calculated using dry silica gel layers for $n$-hexane (C6), $n$-heptane (C7) and $\mathrm{n}$-octane (C8) in magnetic field and outside it.



Figure 5: Values of apolar component of free interfacial energy of silica gel layers determined on the basis of experiments using n-hexane (C6), n-heptane (C7) and n-octane (C8), in magnetic field and outside it. 
Table 1: Values of polar components of free interfacial energy $\left(\nu_{L}^{-}\right.$and $\left.\gamma_{L}^{+}\right)$for water and formamide.

\begin{tabular}{|l|l|l|l|l|}
\hline Liquid & $\boldsymbol{V}_{\mathrm{L}}\left[\mathrm{mN} / \mathrm{m}^{2}\right]$ & $\boldsymbol{V}_{\mathrm{L}}{ }^{\mathrm{LW}}\left[\mathrm{mN} / \mathrm{m}^{2}\right]$ & $\boldsymbol{V}_{\mathrm{L}}{ }^{+}\left[\mathrm{mN} / \mathrm{m}^{2}\right]$ & $\boldsymbol{V}_{\mathrm{L}}\left[\mathrm{mN} / \mathrm{m}^{2}\right]$ \\
\hline Water & 72,80 & 21,80 & 25,50 & 25,50 \\
\hline Formamide & 58,00 & 39,00 & 39,60 & 2,28 \\
\hline
\end{tabular}
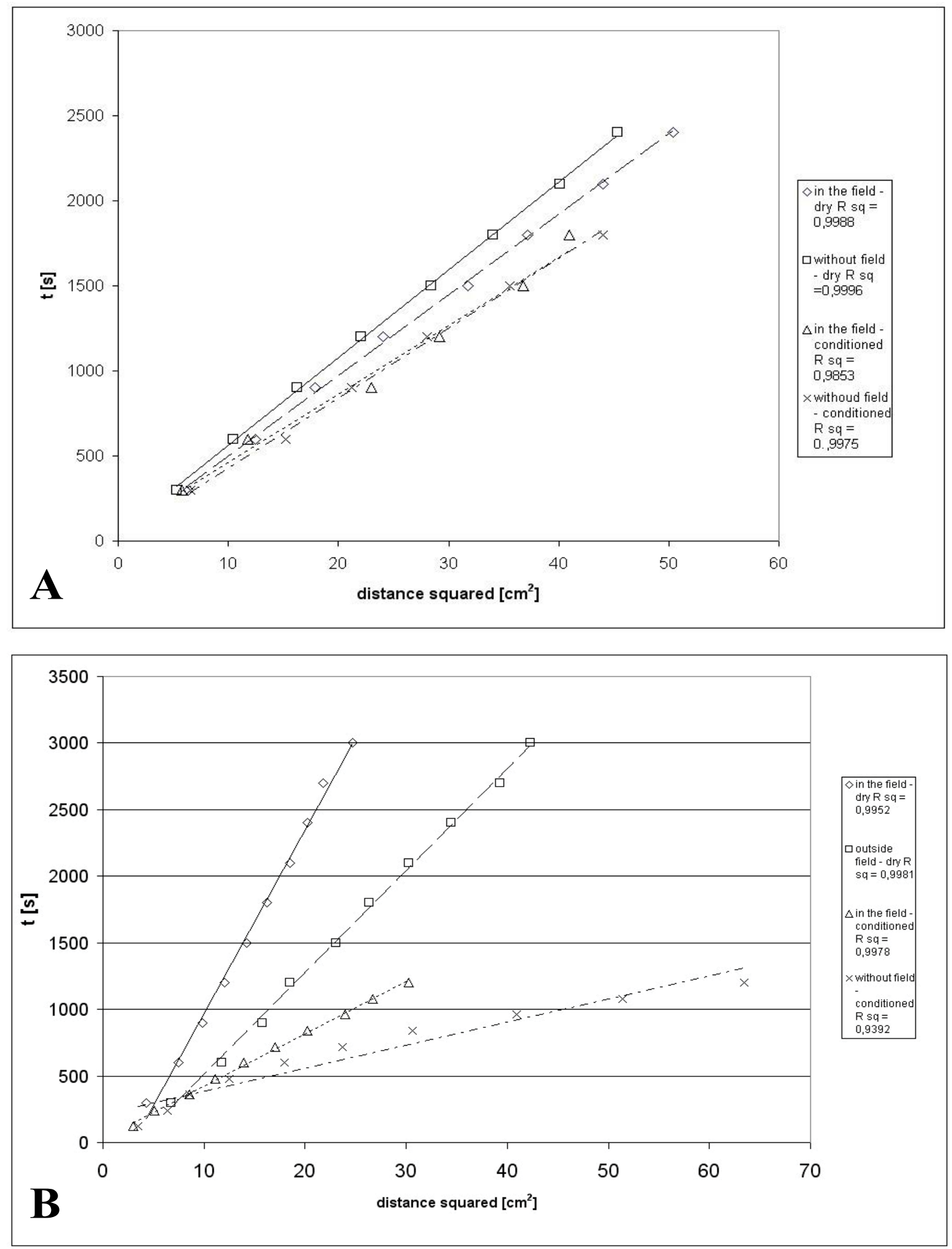

Figure 6: Dependence of migration time of formamide (a) and water (b) on dry and saturated on $\mathrm{SiO}_{2}$ layers in magnetic field and outside it. 
Comparing calculated values outside the field for three consecutive n-alkanes one can observe increase of $\gamma_{s}^{\text {LW }}$ values according to number of carbon atoms in molecule. Analogical comparison for experiment carried out in uniform, perpendicular static magnetic field does not confirm that dependence. Presence of the field raised component value for $n$-hexane, did not change the value calculated for $n$-heptane and decreased it for n-octane.

The cause of that phenomenon can be connected with straightening of $n$-hexane changes in magnetic field what allows to penetrate smaller pores of stationary phase what results increase of contact surface between solid an liquid. In case of $n$-heptane that phenomenon manifests in much weaker way, probably due to odd number of carbons in molecule. For n-octane, decrease of apolar component can be explained in the opposite way as in case of n-hexane. Change of molecule configuration caused by presence of external magnetic field does not allow them to penetrate some pores of solid. Another meaning fact is its lower magnetic permittivity comparing to $\mathrm{n}$-hexane and $\mathrm{n}$-heptane.

\section{Influence of magnetic field on values electron donor $\left(v_{s}^{-}\right)$and electron acceptor $\left(v_{s}^{+}\right)$parame- ters of free interfacial energy}

The values of $\gamma_{s}^{-}$and $\gamma_{s}^{+}$parameters can be calculated on the basis of measurements of solvent front migration velocities on wetted and non wetted layers of two liquids which $\gamma_{L}{ }^{-}$and $\nu_{L}{ }^{+}$are known using following system of equations:

$$
\left\{\Delta G_{b(W)}-\Delta G_{p(W)}=2 \sqrt{\gamma_{S}^{L W} \gamma_{W}^{L W}}+2 \sqrt{\gamma_{S}^{+} \gamma_{W}^{-}}+2 \sqrt{\gamma_{S}^{-} \gamma_{W}^{+}}-2 \gamma_{W}\right.
$$

In this study, water and formamide as test liquids were used. Their ${ }^{-}$and ${\gamma_{L}}^{+}$were found in literature [27] $\Delta G_{p}$ and $\Delta G_{b}$ values were calculated in the same way as in case of n-alkanes used for apolar component determination (Table 1).

Test liquids does not fully spread on the surface of silica gel (they have non-zero static contact angle) thus the spread work of those liquid is equal to difference between $\Delta G_{p}$ and $\Delta G_{b}$. Therefore, in order to determine those values data about solvent front velocities of bipolar test liquids on dry and covered by the liquid films are needed. Obtained experimental data of migration time versus square of distance are presented on Figure 6a and Figure $6 b$.
As it can be seen, in case of water velocity of migration in magnetic field and outside it changes dramatically. In case of formamide changes of migration velocities in magnetic field comparing to water are noticeably smaller. Analysing $\gamma_{L}^{-}$and $v_{L}^{+}$ values of test liquid one may claim that magnetic field influences on electron donor parameter of free surface energy component because in case of formamide (which electron acceptor parameter is dominant) differences between penetration velocity in the magnetic field and outside it are relatively small, and in case of water (in case of which both parameters are equal) differences of penetration time are $\gamma_{L}^{-}$and $\gamma_{L}^{+}$significant.

On the basis of obtained data $\Delta G_{p}$ and $\Delta G_{b}$ values in magnetic field and outside it were calculated (Table 2).

For differences between $\Delta G_{p}$ and $\Delta G_{b}$ for formamide in case of calculations carried out on the basis of $R$ calculated for $\mathrm{n}$-hexane $\mathrm{n}$-heptane and $n$-octane significant influence of magnetic field can be observed. In the field, the value is almost half as big as in case of experiment outside the field. In case of water decrease of those values is also observed but differences are smaller than in case of formamide. These changes do not influence on calculated electron donor and electron acceptor parameters obtained for silica gel. Values of $\gamma_{s}^{-}$and $\nu_{s}^{+}$ calculated on the basis of effective radii interparticle capillaries calculates on the basis of measurements carried out using $n$-hexane, $n$-heptane and $\mathrm{n}$-octane are presented in Table 3.

According to earlier observations, in magnetic field, one may state that electron donor parameter did not changed, but electron acceptor parameter changes were observed. The intensity of changes depends strongly from the type of hydrocarbon used for effective radii interparticle capillaries $R$. The biggest changes of that parameter were observed in $n$-hexane case. In case of $n$-heptane changes are noticeable but much smaller. When $R$ values obtained on the basis of velocity of migration of $n$-octane were used insignificant increase of electron acceptor parameter was observed.

\section{Conclusions}

Presented in this paper experimental data proves that presence of uniform static magnetic field can change values of free interfacial energy in solid-liquid systems even in case of relatively small 
Table 2: Values of $\Delta G_{p}, \Delta G_{b}\left[\mathrm{~mJ} / \mathrm{m}^{2}\right]$ and differences between them calculated on the basis of values of effective radii interparticle capillaries $(R)$ calculated for $n$-hexane, $n$-heptane and $n$-octane in magnetic field for formamide and water.

\begin{tabular}{|c|c|c|c|c|}
\hline & \multicolumn{2}{|l|}{ Formamide } & \multicolumn{2}{|l|}{ Water } \\
\hline & $\begin{array}{l}\text { In magnetic } \\
\text { field }\end{array}$ & $\begin{array}{l}\text { Outside magnetic } \\
\text { field }\end{array}$ & $\begin{array}{l}\text { In magnetic } \\
\text { field }\end{array}$ & $\begin{array}{l}\text { Outside magnetic } \\
\text { field }\end{array}$ \\
\hline \multicolumn{5}{|c|}{ R n-hexane } \\
\hline$\Delta G_{p}$ & 3,56 & 3,52 & 0,99 & 1,12 \\
\hline$\Delta G_{b}$ & 3,15 & 2,75 & 0,335 & 0,45 \\
\hline$\Delta G_{b}-\Delta G_{p}$ & $-0,41$ & $-0,77$ & $-0,66$ & $-0,68$ \\
\hline \multicolumn{5}{|c|}{ R-n-heptane } \\
\hline$\Delta G_{p}$ & 3,11 & 3,35 & 0,87 & 1,07 \\
\hline$\Delta G_{b}$ & 2,75 & 2,62 & 0,29 & 0,43 \\
\hline$\Delta G_{b}-\Delta G_{p}$ & $-0,36$ & $-0,73$ & $-0,58$ & $-0,64$ \\
\hline \multicolumn{5}{|c|}{ R-n-octane } \\
\hline$\Delta G_{p}$ & 3,36 & 3,58 & 0,94 & 1,14 \\
\hline$\Delta \mathrm{G}_{\mathrm{b}}$ & 2,98 & 2,80 & 0,31 & 0,46 \\
\hline$\Delta G_{b}-\Delta G_{p}$ & $-0,39$ & $-0,78$ & $-0,63$ & $-0,69$ \\
\hline
\end{tabular}

Table 3: Values of electron donor $\left(\gamma^{-}\right)$and electron acceptor $\left(\gamma^{+}\right)$parameters calculated on the basis of $R$ values determined for $n$-hexane, $n$-heptane and $n$-octane in magnetic field $\left(v_{(m)}\right)$ and outside it () .

\begin{tabular}{|c|c|c|c|c|c|c|}
\hline & $\gamma_{s(m)}^{-}$ & $v_{s}^{-}$ & $\gamma_{s(m)}^{-}-\gamma_{s}^{-}$ & $V_{s(m)}^{+}$ & $\mathrm{V}_{\mathrm{s}}^{+}$ & $V_{s(m)}^{+}-V_{s}^{+}$ \\
\hline n-hexane & 54,6 & 53,6 & 1,00 & 3,3 & 5,2 & $-1,9$ \\
\hline n-heptane & 54,4 & 54,0 & 0,4 & 3,7 & 3,8 & $-0,1$ \\
\hline n-octane & 54,7 & 54,4 & 0,3 & 3,0 & 2,7 & 0,3 \\
\hline Mean & 54,6 & 54,5 & 0,6 & 3,33 & 3,9 & $-0,4$ \\
\hline
\end{tabular}

inductivities of that field. The values of all parameters describing components of free interfacial energy in the area of field presence were different from those obtained in the area where the field was not present. Regarding that all other experimental parameters in the field and outside it were exactly identical, existence of differences can be assigned to the fact of presence the external uniform static field. What supports the theses suggested earlier works regarding influence of physical fields on properties of matter and interfacial phenomena under the influence of magnetic field $[23,25,26,28,29]$.

\section{Acknowledgements}

The experiment presented in this paper was a part of project "Development of the JINR Basic Facility for Generation of Intense Heavy Ion and Polarized Nuclear Beams Aimed at Searching for the
Mixed Phase of Nuclear Matter and Investigation of Polarization Phenomena at the Collision Energies to $\sqrt{S N N}$ up to $=11 \mathrm{GeV}$ - Influence of magnetic field on interactions of chosen solutes with different surfaces by use planar chromatographic (TLC) method."

This work is dedicated in memoriam of Dr. Henryk Malinowski a beloved husband and excellent fellow scientist who unexpectedly died before publication of this paper.

\section{References}

1. I Malinowska, M Studziński, H Malinowski (2008) The effect of a magnetic field on the retention of polyaromatic hydrocarbons in planar chromatography. J Planar Chromat 21: 379-385.

2. I Malinowska, M Studziński, H Malinowski (2011) 
Some aspects of TLC in homogenous magnetic fields. J Sep Sci 34: 1788-1795.

3. I Malinowska, M Studziński, H Malinowski, M Gadzikowska (2017) Retention and separation changes of ternary and quaternary alkaloids from Chelidonium majus L. by TLC under the influence of external magnetic field. Chromatographia 80: 923-930.

4. FM Fowkes (1962) Determination of interfacial tensions, contact angles and dispersion forces in surfaces by assuming additivity of intermolecular interactions in surfaces. J Phys Chem 66: 382-382.

5. CJ van Oss, RJ Good, MK Chandhury (1986) The role of van der Waals forces and hydrogen bonds in "hydrophobic interactions" between biopolymers and low energy surfaces. J Colloid Interface Sci 111: 378390.

6. CJ van Oss, RJ Good, MK Chandhury (1988) Interfacial Lifshitz-van der waals and polar interactions in macroscopic systems. Chem Rev 88: 927-941.

7. CJ van Oss, RJ Good, MK Chandhury (1992) The modern theory of contact angles and the hydrogen bond components of surface energies. In: ME Schrader, GI Loeb, Modern approach to wetability: Theory and applications.

8. LA Girifalco, RJ Good (1957) A theory for estimation of surface and interfacial energies. I. Derivation and application to interfacial tension. J Phys Chem 64: 904-904.

9. IM Lifshitz, AM Kossevich, Ya E Geguzin (1967) Surface phenomena and diffusion mechanism of the movement of defects in ionic crystals. J Phys Chem Solids 28: 783-792.

10.PA Small (1953) Some factors affecting the solubility of polymers. J Appl Chem 3: 72-80.

11.PA Kolmann, J McKevley, A Johanson, S Rothenberg (1975) Theoretical studies of hydrogen-bonden dimers - complexes involving $\mathrm{HF}, \mathrm{H} 2 \mathrm{O}, \mathrm{NH} 3, \mathrm{HCL}, \mathrm{H} 2 \mathrm{~S}$, $\mathrm{PH} 3, \mathrm{HCN}, \mathrm{HNC}, \mathrm{HCP}, \mathrm{CH} 2 \mathrm{NH}, \mathrm{H} 2 \mathrm{CS}, \mathrm{H} 2 \mathrm{CO}, \mathrm{CH} 4$, $\mathrm{CF} 3 \mathrm{H}, \mathrm{C} 2 \mathrm{H} 2, \mathrm{C} 2 \mathrm{H} 4, \mathrm{C} 6 \mathrm{H} 6, \mathrm{~F}-, \mathrm{AND} H 3 \mathrm{O}+$. J Am Chem Soc 97: 955-965.

12.PA Kolmann (1977) General analysis of noncovalent intermolecular interactions. J Am Chem Soc 99: 4875-4894.

13.RJ Good, LA Girifalco (1957) A theory for the estimation of surface and interfacial energies. I. Derivation and application to interfacial tension. J Phys Chem 62: 904-909.

14.RJ Good, LA Girifalco (1960) A theory for estimation of surface and interfacial energies. III. Estimation of surface energies of solids from contact angle data. J Phys Chem 64: 561-565.

15.FM Fowkes (1964) Attractive forces at interfaces. J Ind Eng Chem 56: 40-52.

16.E Chibowski, F Gonzales-Caballero (1993) Theory and practice of thin layer wicking. Langmuir 9: 330-340.

17.E Hibowski, L Hołysz, A Szcześ (2017) Application of thin-layer wicking method for surface free energy determination. Surface Innovations 5: 9-20.

18. L Hołysz (1994) Studies or surface-free energy of celestite. Polish J Chem 68: 2699-2705.

19.L Hołysz, E Chibowski (1992) Surface free energy components and flotability of barite precovered with sodium dodecyl sulfate. Langmuir 8: 303-308.

20.RF Giese, PM Constanzo, CJ van Oss (1991) The surface free-energies of ttalc and pyrophyllite. Phys Chem Miner 17: 611-616.

21.E Chibowski, L Hołysz (1997) On the use of Washbourn equation for contact angle determination. J Adhesion Sci Technol 11: 1289-1301.

22.LD Leslie-Pelecky, RD Rieke (1996) Magnetic properties of nanostructured materials. Chem Mater 8: 1770-1783.

23.LP Oommen, GN Kumar (2019) A study on the effect of magnetic field on the properties and combustion hydrocarbon fuels. Int J Mech Prod Eng Res Dev 9: 89-98.

24.S Ueno, M Iwasaka (1994) Properties of diamagnetic fluid in high-gradient magnetic fields. J Appl Phys 75: 7177-7179.

25. Y Wang, B Zhang, Z Gong, K Gao, Y Ou, et al. (2013) The effect of a static magnetic field on the hydrogen bonding in water using frictional experiments. J Mol Struct 1052: 102-104.

26. R Cai, H Yang, J He, W Zhu (2009) The effects of magnetic fields on water molecular hydrogen bonds. J Mol Struct 938: 15-19.

27. CJ van Oss, RJ Good (1989) Surface tension and the solubility of polymers and biopolymers: The role of polar and apolar interfacial free energies. J Macromol Sci Part A: Pure Appl Chem 26: 1183-1204.

28.Y Fujimura, M lino (2009) Magnetic field increases the surface tension of water. J Physics: Conference Series 156: 012028.

29.E Hibowski, A Szcześ (2018) Magnetic water treatment, review of latest approaches. Chemosphere 203: 54-67. 